\title{
Haavelmo's Epistemology for an Inexact Science
}

\author{
Marcel Boumans
}

Haavelmo remarks that physicists are very clever. They confine their predictions to the outcomes of their experiments. They do not try to predict the course of a rock in the mountains and trace the development of the avalanche. It is only the crazy econometrician who tries to do that, he says. -Nancy Cartwright, The Dappled World: A Study of the Boundaries of Science (1999)

Historians of econometrics generally agree on the paradigmatic role Trygve Haavelmo's The Probability Approach in Econometrics (1944) had in creating modern econometrics. For example, Mary Morgan's History of Econometric Ideas calls the methodology presented in The Probability Approach the "blueprint for econometrics" (Morgan 1990, 251). When historians do so, however, they usually refer to the second part of Haavelmo's work, where the "Neyman-Pearson theory of testing statistical hypotheses" (Haavelmo 1944, v) is introduced to econometrics, and hardly ever to its first two chapters, "Abstract Models and Reality" and "The Degree of Permanence of Economic Laws." An early review by Edwin B. Wilson $(1946,173)$ is representative of the general reception of those two chapters:

Correspondence may be addressed to Marcel Boumans, University of Amsterdam, Department of Economics, Roetersstraat 11, 1018 WB Amsterdam, The Netherlands; e-mail: M.J.Boumans @uva.nl. I would like to thank Kevin D. Hoover for his constructive support.

History of Political Economy 46:2 DOI 10.1215/00182702-2647477

Copyright 2014 by Duke University Press 
The work is difficult reading. ... The author's approach is extremely abstract and metaphysical; [the first two chapters seem] to be written quite as much from the point of view of the philosophy of scientific method in general as from that of economic analysis in particular.

Ignoring the negative tone of the review, I fully agree with Wilson. Haavelmo's first two chapters provide a very rich epistemological framework for understanding what it entails to do scientific research as a "passive observer," that is, when unable to conduct experiments "to see if certain real economic phenomena-when artificially isolated from "other influences'-would verify certain hypotheses" (Haavelmo 1944, 14).

This article aims to clarify Haavelmo's epistemology presented in the first two chapters of his Probability Approach, by providing the historical context from which it arose. In the foreground of this history is the shared research agenda of "scientization" (Bjerkholt and Qin 2011, 2) by Ragnar Frisch, Jan Tinbergen, and Tjalling Koopmans; more in the background is a longer discussion, started in the nineteenth century, of whether and in what sense economics can be considered an "exact science."

\section{Whether Economics Is an Exact Science}

Except these first two chapters of The Probability Approach, there are no other publications of Haavelmo in which he discusses his epistemology explicitly. Similarly, Haavelmo's mentor and supervisor Ragnar Frisch never gave an explicit enough view of his conception of science. In a series of lectures he gave at Yale University in the fall of 1930, ${ }^{1}$ however, Frisch opened his first lecture with a discussion of the terms science and scientific by taking astronomy as the exemplary "true science":

Astronomy is a field of study which is "scientific" more than any other of the fields of study having as their object the exploration of the exterior world. The reason for this, it seems, is that in astronomy the fusion of theory and observation has been realized more perfectly than in the other fields of study. When astronomy is a science, it is not because it has an abstract theoretical structure, nor it is because it is built on minute prolonged observations, but it is because the astronomical observations are filled into the theoretical structure. (Frisch [1930] 2011, 29)

1. Recently edited by Olav Bjerkholt and Duo Qin (2011) and characterized as "teaching econometrics as a science" (1). 
Frisch continued his first lecture by remarking that economics had not yet reached the stage of astronomy, where theory and observations had been unified. Even though economics had accumulated "overwhelming statistical and historical material on economic facts," those observations had "not been guided and animated by constructive theoretical thinking in the same way as the astronomical observations" (29).

This view on what "true science" is and relating it to astronomy has a long tradition in economics, where we can take John Stuart Mill's statement in A System of Logic (first published in 1843), "that there is, or may be, a science" of economics, as a starting point. Mill ([1843] 1911, 553) did not regard economics as an "exact science." The basic premises of economics state accurately how specific causal factors operate, but, according to Mill, they are statements of tendencies and are inexact rather than universal generalizations. Economists know the major causes of economic phenomena, but there are many "inferences" or "disturbing causes."

In an exact science such as astronomy, explanation is complete: "The greater causes, those on which the principal part of the phenomenon depends," Mill writes, "are within the reach of observation and measurement; so that if no other causes intervened, a complete explanation could be given not only of the phenomenon in general, but of all the variations and modifications which it admits of" (552). A science is exact when "its phenomena have been brought under laws comprehending the whole of the causes by which the phenomena are influenced, whether in a great or only in a trifling degree, whether in all or only in some cases, and assigning to each of those causes the share of effect which really belongs to it" (553).

In opposition to this, in an inexact science

the only laws as yet accurately ascertained are those of the causes which affect the phenomenon in all cases, and in considerable degree; while others which affect it in some cases only, or, if in all, only in a slight degree, have not been sufficiently ascertained and studied to enable us to lay down their laws, still less to deduce the completed laws of the phenomenon, by compounding the effects of the greater with those of the minor causes. (553)

Mill refers to the science of tides as an example of an inexact science. Scientists know the laws of the great causes, the gravitational attraction of the sun and the moon, but they are ignorant of "circumstances of a local or casual nature, such as the configuration of the bottom of the ocean, the degree of confinement from shores, the direction of the wind, \&c" (553). 
Since economists, in Mill's view, know only the laws of the "greater causes" of the phenomena, they are unable to infer invariably and precisely what actually occurs. Economic theory considers only some of the "great causes" of economic phenomena. Economics is in this way an inexact science. The inability to infer what actually happens is a consequence of inexact theories, not merely of faulty data or mathematical limitations.

About forty years later, Alfred Marshall ([1890] 1930, 32) made the same comparison of economics with the science of tides, to discuss economics as an inexact science:

The laws of economics are to be compared with the laws of the tides, rather than with the simple and exact law of gravitation. For the actions of men are so various and uncertain, that the best statement of tendencies, which we can make in a science of human conduct, must needs be inexact and faulty.

Marshall devoted chapter 3 (of book 1) of the Principles to a discussion of the nature of "economic generalizations or laws." Why, he asked, should the "laws of economics" be less predictable and precise in their workings than the law of gravitation? The key to Marshall's view lies in his claim that economic mechanisms work out their influences against a messy background of complicated factors, so that the most we can expect of economic analysis is that it captures the "tendencies" induced by changes in this or that factor.

No one knows enough about the weather to be able to say beforehand how it will act. A heavy downpour of rain in the upper Thames valley, or a strong north-east wind in the German Ocean, may make the tides at London Bridge differ a great deal from what had been expected. (32)

Like Mill, Haavelmo $(1944,40)$ did not believe that economics could be turned into an exact science such as astronomy.

From experience we know that attempts to establish exact functional relationships between observable economic variables would be futile. It would indeed be strange if it were otherwise, since economists would then find themselves in a more favorable position than any other research workers, including the astronomers. Actual observations, in whatever field we consider, will deviate more or less from any exact functional relationship we might try to establish. 
As an "apprentice" of Frisch (Bjerkholt 2005), he was too much aware of the limitations of the use of statistics only, as taught by Frisch:

I do not claim that the technique developed in the present paper will, like a stone of the wise, solve all the problems of testing "significance" with which the economic statistician is confronted. No statistical technique, however refined, will ever be able to do such a thing. The ultimate test of significance must consist in a network of conclusions and cross checks where theoretical economic considerations, intimate and realistic knowledge of the data and a refined statistical technique concur. (Frisch 1934, 129)

Two other pioneers of econometrics, Jan Tinbergen and Tjalling Koopmans, the Dutch compeers of Frisch and Haavelmo, also recognized that statistics is not sufficient by itself to make something an exact science.

\section{Linear Regression Analysis in the 1930s}

The awareness that by statistical techniques only-at that time mainly linear regression analysis - one could never arrive at a complete list of causal factors was part of the common understanding of what one could do with statistics among these four men in the late 1930s. Koopmans's dissertation, "Linear Regression Analysis of Economic Time Series," is exemplary in this regard. It was published as a Netherlands Economic Institute (NEI) publication in 1937. In the preface, the directors of the $\mathrm{NEI}^{2}$ justified their decision to publish it by explaining the methodological problem one has to deal with in economics in contrast to a laboratory science:

One of the great difficulties in the statistical testing of economic laws is the famous "ceteris paribus" clause with which theory has to be formulated. The statistician wishing to isolate a given law has to eliminate changes of these other factors assumed to be constant in that law. The most effective implement for this sort of problem is the method usually called multiple correlation analysis, which method has been applied in several investigations in the last few years. It is still an open question,

2. At that time, the board of directors consisted of P. Lieftinck, N. J. Polak, J. Tinbergen, and F. de Vries. 
however, whether the results thus obtained have real significance and, if so, to what degree the figures calculated are trustworthy. (Koopmans 1937, v)

To deal with the impossibility of keeping everything else the same, Koopmans suggests the following division of labor between the "economist" and the "statistician." The economist "should by economic reasoning and general economic experience-or by his knowledge of the special branch of science concerned-devise a set of determining variables which he expects to be a complete set" (57). A set $x_{1}, x_{2}, \ldots, x_{n}$ is defined to be complete if the combined influence on $y$ of all other variables not included in the set is represented by a relatively "small summand" in $y$ of an "accidental nature." "If, in any concrete situation, a standard is established for what should be understood by a 'small summand of accidental nature,' the notion of a complete set of determining variables is fixed by that standard" (6).

The statistician, however, cannot test the completeness of this set. "The possibility remains that the supposed complete set, though differing from the real one in at least one variable, figures in the regression equation as a representative of it, and is able to do so because of close interrelations, 'by chance' or otherwise conditioned, between variables of the two sets" (57). In order to decide what may occur "by chance," Koopmans saw that the statistician's task requires knowledge of the laws governing the behavior of the variables concerned.

Although the statistician cannot confirm that the economist has correctly identified the complete set of relevant variables or the laws underlying their behavior, in some cases she may be able to show that the economist is wrong-namely, in those cases in which a regression equation "expressing the dependent variable in terms of the determining variables of the supposed complete set, fitted to the data, leaves large residuals" (58). In the case of small residuals which do not exhibit systematic variation,

the task of the statistician is confined to a study of the reliability of the empirical regression coefficients on the hypothesis that the economist indicated the right set of determining variables, or, at least, that he did not omit important determining variables from his list. Such a study is, indeed, possible. (58)

The task of the statistician was modest, in the sense that it was restricted to an evaluation of the reliability of the regression coefficients and to 
telling the economist whether she had omitted a causal factor, but not what the omitted factor was.

\section{Haavelmo's Critique}

Haavelmo, being acquainted with the latest developments in econometrics, was studying what it meant for a "determining variable" to be "important." ${ }^{3}$ In this period - the late 1930s and early 1940s - he was clearly investigating that issue, witnessed by three papers written in the period and that will be discussed here.

In a lecture given at the Third Nordic Meeting of Younger Economists, in Copenhagen in May 1939, Haavelmo ([1939] 2008, 14) remarked that if there is a "statistical agreement" between the observed data and the regression equation, it is still uncertain whether the regression coefficients are "significant," that is, whether the absolute values of the coefficients are large relative to their standard errors.

Haavelmo may have received this specific idea of "significance" when he visited Tinbergen in Geneva, when Tinbergen was at the League of Nations and whose research there led to the two-volume Statistical Testing, published in 1939. There is some evidence that Tinbergen, in turn, got the idea from Ragnar Frisch (Boumans 2010). There is, however, so far no evidence that Haavelmo got that idea directly from Frisch.

Tinbergen defined the term $b_{2} x_{2}$ in an expression like $y=b_{2} x_{2}+b_{3} x_{3}$ $+\ldots$, as the "influence of $x_{2}$." The "strength of this influence" was defined as $b_{2} \sigma_{2}$, that is, the standard deviation of $x_{2}$ times the regression coefficient (Tinbergen 1939a, 22-23).

To discuss whether this "strength of influence" could play a role in evaluating whether a causal factor was relevant, Haavelmo ([1939] 2008) made a distinction between the "error of the momentary explanation" and the "error of the average relation."

He showed the difference between these two with the following example: Consider $N$ observations on three variables, $x, y$, and $z$, between which exists the following true but unknown relation (note that the three variables are measured from their respective means):

3. Bjerkholt (2005) suggests that Tjalling Koopmans's visit to Oslo may have had a lasting influence ("a point of no return") on Haavelmo. Koopmans spent the autumn of 1935 at the University Institute of Economics and gave a series of lectures on "modern sampling theory," discussing Fisher's theory of estimation and the Neyman-Pearson theory of hypothesis testing. Haavelmo (1938) reviewed Koopmans's (1937) dissertation. 
218 History of Political Economy 46:2 (2014)

$$
y=2 x-3 z
$$

Suppose we assume that a relationship exists between $y$ and $x$ but we are ignorant about the influence of $z$ :

$$
y=b x .
$$

The correlation coefficient between $y$ and $x$ is

$$
r_{x y}=2 \frac{\sigma_{x}}{\sigma_{y}}-3 r_{x z} \frac{\sigma_{z}}{\sigma_{y}}
$$

As a result, the regression coefficient $b$ is

$$
b=2-3 r_{x z} \frac{\sigma_{z}}{\sigma_{x}}
$$

Haavelmo defined the "error of the average relation" as the standard error of the regression coefficient $b$ :

$$
\sigma_{b}=\frac{1}{\sqrt{N-2}} \frac{\sigma_{y}}{\sigma_{x}} \sqrt{1-r_{x y}^{2}} .
$$

This error tells us that, despite the omission of $z$, the larger the number of observations, the more precisely the regression between $y$ and $x$ is determined. So, according to Haavelmo, the larger the $N$, the "more stable" the "average relation" between $y$ and $x$. But this does not mean that this relation is "a good description of the momentary variation in $y$ " (Haavelmo [1939] 2008, 15).

He defined the "error of the momentary relation" 4 as the mean squared difference between the observed $y$ and the estimated, called the "calculated," $\hat{y}$ :

$$
\frac{1}{N} \sum_{i=1}^{N}\left(y_{i}-\hat{y}_{i}\right)^{2}=\frac{1}{N} \sum_{i=1}^{N}\left(2 x_{i}-3 z_{i}-2 x_{i}+3 r_{x z} \frac{\sigma_{z}}{\sigma_{X}} x_{i}\right)^{2}=9\left(1-r_{X Z}^{2}\right) \sigma_{z}^{2} .
$$

So, when the number of observations increases, the "error in the average explanation," $\sigma_{b}$, decreases, but the "error in the momentary explanation" remains at the same level of $9\left(1-r_{x z}^{2}\right) \sigma_{z}^{2}$.

This "error in the momentary explanation" was subsequently used to discuss the following cases: The smaller the variation in $z, \sigma_{z}$, and the

4. Like Frisch, Haavelmo had the habit of coining new terms in every next paper while actually discussing the same issues. Without being able to provide more textual evidence, I assume in the present paper that Haavelmo used "average equation" and "confluent equation" (in his other papers) as synonyms. In the same way, I assume that "momentary equation" is synonymous with "autonomous equation." 
larger the correlation $r_{x z}$, the better the "momentary explanation of $y$." A high correlation $r_{x z}$ means that the variation in $x$ also accounts for part of the variation in $z$, so the omission of $z$ is not that bad. The more interesting case, however, is the opposite case, when both the correlation $r_{x z}$ and the variation of $z, \sigma_{z}$, are small:

This means that $z$ is a superfluous variable when it comes to explaining the observed variation of $y$ in this material. But that does not necessarily mean that [the regression equation] will give a good forecast of $y$ outside the period covered by the data. (Haavelmo [1939] 2008, 15)

Equation (1) shows that if $z$ varies strongly enough, it would exert a substantial influence. So, although $x$ alone may give a good explanation of $y$ for the used data set, "it may be of decisive importance whether or not we can utilize the tiny part of the variation remaining in attempting to capture the effect of $z$ " (15-16).

This indeed shows how crucial it is to have, in advance, a formulation of the hypotheses, in which one operates with specific fictitious variations. If one refrains from doing so, one runs the risk of missing out important variables that, by change or for specific reasons, have not shown significant variation in the material at hand. And although a simpler hypothesis may give a stable average explanation and for that reason gets accepted as statistically valid, it may well give a very poor, maybe a completely worthless momentary explanation and no deeper insight into structural relationships between the studied variables. (16)

In other words, the "strength of the influence," that is, the regression coefficient times the standard deviation, is not a good indicator of whether a variable is a deeper, structural ("autonomous") factor, because it depends too much on whether the variable has shown significant variation.

This same issue was also discussed in a more direct criticism of Tinbergen's method employed in his work at the League of Nations. In a 1941 note, Haavelmo (1941a) criticizes Tinbergen's method of using regression analysis to select the main causal factors, in particular Tinbergen's decision that the rate of interest does not causally affect investment.

According to the "classical dynamic theories of production and prices," the interest rate is a "powerful autonomous parameter" (Haavelmo 1941a, 49). But Tinbergen's (1939a) regression analysis shows that the regression coefficient of the interest rate is not significantly different from zero, and therefore Tinbergen concludes that "variations in the rate of interest play 
only a minor role, or no role at all, in the changes in investment activity" (Haavelmo 1941a, 49). Haavelmo's note, however, aims to show that the coefficient's being zero "by itself is not sufficient to establish this conclusion" (49). Moreover, it aims to show that regression equations "may be very misleading if the regression coefficients [are] taken to represent effects of autonomous changes in the corresponding variables" (49).

To better understand what Haavelmo's criticism entails, it first should be noted that Tinbergen did not only use the "influence" of a factor for evaluating whether a variable was relevant but also the "strength of the influence" (Boumans 2010). In his "statistical testing," the strength of the interest rate showed this influence to be "far less important than profits and share yields" (Tinbergen 1939a, 55).

Haavelmo showed with a model representing the structural relationships with respect to investment activity that even if the interest rate is a "causal component," its regression coefficient can still be zero: Let $y_{1}$, $y_{2}, \ldots, y_{n}$ be a number of causal components, where for this case $y_{1}$ is gross profit, $y_{2}$ is total interest expenses, etc. Let $Y, x_{1}, x_{2}, \ldots, x_{n}$ be the observable variables, where for this case $Y$ is the volume of investment $(v)$, $x_{1}$ is net profit $(z), x_{2}$ is the interest rate $(m)$, etc. The regression equation which should fit the data is

$$
v=k_{1} z+k_{2} m+\ldots+\varepsilon .
$$

But if

$$
\begin{aligned}
& v=k\left(y_{1}+y_{2}+\ldots+y_{n}\right)+\varepsilon \\
& z=a_{1}\left(y_{1}+y_{2}+\ldots+y_{n}\right) \\
& m=a_{2} y_{2},
\end{aligned}
$$

then the regression coefficient $k_{2}$ is zero. But also when $m=a_{3} y_{4}$, where $y_{4}$ is "some other effects of the interest on investment, which are not included in the calculation of net profit" (Haavelmo 1941a, 50), like the substitution effect, different preference schedules for different kinds of holdings, etc., $k_{2}$ would still be zero, because these effects are partly taken care of by net profit. This result (that $k_{2}$ would still be zero), however, as Haavelmo emphasized, does not imply that changes in the interest rate have no influence upon investment; the interest rate "may indirectly exercise a decisive influence upon $v$, namely via its influence upon $z$ " (50).

Besides the fact that the causal structure can be such that the regression coefficient of a causal component will always be zero, Haavelmo gave a second reason for the coefficient being zero: 
The rate of interest may not have varied much during the statistical testing period, and for this reason the rate of interest would not "explain" very much of the variation in net profit (and thereby the variation in investment) which has actually taken place during this period. But one cannot conclude that the rate of interest would be inefficient as an autonomous regulator, which is, after all, the important point. (50)

This point was acknowledged by Tinbergen $(1939 b, 13)$ in the second volume of Statistical Testing:

It goes without saying that if some explanatory factor has not changed at all in the period studied, its influence cannot be determined. If it changed only slightly, its regression coefficient may be uncertain. Extrapolation of such results for large variations in the factors concerned is therefore not permitted.

\section{Haavelmo's Epistemology}

The epistemological insights of the 1939 Copenhagen lecture and his note on the interest rate were generalized in his 1941 treatise On the Theory and Measurement of Economic Relations, which was published in 1944 as The Probability Approach in Econometrics. In particular, the section titled "The Question of Simplicity in the Formulation of Economic Laws" discusses the problem of arriving at a complete explanation of certain economic behaviors or phenomena. ${ }^{5}$ This general framework allowed him to discuss issues about finding laws outside ceteris paribus environments.

Haavelmo did not share Mill's pessimism: "A phrase such as 'In economic life there are no constant laws,' is not only too pessimistic, it also seems meaningless" (Haavelmo 1944, 14). Instead, Haavelmo suggested that "we may try to find a rational explanation for the fact that relatively few attempts to establish economic "laws' have been successful" (14).

The "rational explanation" he developed in his dissertation can be clarified by first considering the following generalization of his analysis presented in the Copenhagen lecture. Note again that the variables are measured from their respective means. Assume that the true relation between $y$ and a set of independent causal factors (correlation coefficients between them are zero) is

5. Section 10, pp. 33-39, in the 1941 version, and section 7, pp. 21-26, in the 1944 version. Both versions are exactly the same. Page numbers refer to the 1944 publication. 


$$
y=a_{1} x_{1}+a_{2} x_{2}+\ldots+a_{n} x_{n}
$$

Assume that one of the variables, say, $x_{i}$, does not deviate from its mean $\left(\sigma_{i}=0\right)$, that is, it remains constant for the considered data set. Then, the correlation of variable $x_{i}, r_{i y}=a_{i} \sigma_{i} / \sigma_{y}=0$ and thus the regression equation is

$$
\hat{y}=a_{1} x_{1}+\ldots+a_{i-1} x_{i-1}+a_{i+1} x_{i+1}+\ldots+a_{n} x_{n} .
$$

As a result, the "error of the momentary explanation" is

$$
\frac{1}{N} \sum_{k=1}^{N}\left(y_{k}-\hat{y}_{k}\right)^{2}=a_{i}^{2} \sigma_{i}^{2}
$$

In his later assessment of this result, Haavelmo (1944) saw that this "error" could be reinterpreted as a measure of the "factual influence" of the variable $x_{i}$ on $y$, which he defined as follows: Let $y$ be a theoretical variable defined as a function of $n$ independent causal variables $x_{1}$, $x_{2}, \ldots, x_{n}$ :

$$
y=f\left(x_{1}, x_{2}, \ldots, x_{n}\right) .
$$

Let us replace the variable $x_{i}$ by a constant $c_{i}$ so determined that

$$
Q_{1}=\sum_{j=1}^{N}\left[f\left(x_{1 j}, x_{2 j}, \ldots x_{i j}, \ldots x_{n j}\right)-f\left(x_{1 j}, x_{2 j}, \ldots c_{i}, \ldots x_{n j}\right)\right]^{2}
$$

is a minimum with respect to $c_{i}$. The factual influence of the variable $x_{i}$ upon $y$ is then defined as Constant $\cdot \sqrt{Q_{i}^{(\min )}}$.

To see that the factual influence is indeed a generalization of the error in the momentary explanation, take the following linear case: $f\left(x_{1}, x_{2}, \ldots\right.$, $\left.x_{n}\right)=a_{1} x_{1}+a_{2} x_{2}+\ldots+a_{n} x_{n}$. Then $c_{i}=\bar{x}_{i}$ and thus $Q_{i}^{(\min )}=N a_{i}^{2} \sigma_{i}^{2}$.

To arrive at a more general framework to discuss the relevance of causal factors, Haavelmo $(1944,23)$ defined the "potential influence" of a causal factor $x_{i}$ upon $y$ as

$$
\Delta_{i} y=f\left(x_{1}, x_{2}, \ldots, x_{i}+\Delta x_{i}, \ldots, x_{n}\right)-f\left(x_{1}, x_{2}, \ldots, x_{n}\right) .
$$

To compare the size of the potential influence of each of the variables $x_{i}$, one has, for any point $\left(x_{1}, x_{2}, \ldots, x_{n}\right)$, to choose a set of displacements $\Delta x_{1}, \Delta x_{2}, \ldots, \Delta x_{n}$, which are considered to be of "equal size according to some standard of judgment" (23).

This concept of potential influence can be clarified by using the definition of a partial derivative:

$$
\frac{\partial f}{\partial x_{i}} \approx \frac{f\left(x_{1}, x_{2}, \ldots, x_{i}+\Delta x_{1}, \ldots, x_{n}\right)-f\left(x_{1}, x_{2}, \ldots, x_{i}, \ldots, x_{n}\right)}{\Delta x_{i}} .
$$


For a fixed set of displacements, say, $\Delta x_{i}=\Delta_{i}$, the potential influence can be rewritten as

$$
\Delta_{i} y=\frac{\partial f}{\partial x_{i}} \Delta_{i}
$$

As we can infer from this expression, the potential influence is a feature of the function $f$, or as Haavelmo put it, "For a given system of displacements $\Delta x_{1}, \Delta x_{2}, \ldots, \Delta x_{n}$, the potential influences are, clearly, formal properties of the function $f$ " (23-24).

In the Copenhagen lecture Haavelmo made a few remarks that confirm my interpretation of potential influence in terms of partial derivatives. In a section on the ceteris paribus clause, he distinguishes between two types of ceteris paribus clauses. The first is the usual type where in the selection of essential "elements" guided by theory and data, one imposes the ceteris paribus clause on all remaining unspecified elements, "because the total effect of these other elements has by experience played no large part for the problem at hand and neither can be expected to do so in the future, so that whether we assume them unchanged or let them play freely is virtually of no consequence" (Haavelmo [1939] 2008, 8). This will be discussed below. The second type, of more interest for the interpretation of "potential influence," is

the one we impose within the system of specified variables. The idea here is just the same as that of partial derivatives. We study relations between some among the specified objects, which are mutually independent, subject to the assumption that the remaining elements specified are kept constant. Usually, the form that such relations takes depends on the level at which the other elements are fixed. Such reasoning is not only of theoretical interest, on the contrary, it is also the basis for assessing effects of practical measures of intervention in the economic activity. (8)

This latter aspect of assessing the effects of interventions with partial derivatives will be discussed below.

Using the expression for potential influence, we can now also rewrite the expression for the factual influence as ${ }^{6}$

$$
\frac{\partial f}{\partial x_{i}} \Delta x_{i}
$$

6. See Boumans 2010 for the derivation of this expression. 
In other words, the factual influence consists of two components: potential influence and variation. The magnitude of the first component is only known if the second component (that is, the variation) is significantly different from zero.

With these two concepts (potential influence and factual influence), Haavelmo could now better clarify the limits of statistical inference. In economics, the investigator is a "passive observer," which means that "he is not in a position to enforce the prescriptions of his own designs of ideal experiments" (Haavelmo 1944, 7). The problem then is that with respect to finding and verifying laws,

how far do the hypothetical "laws" of economic theory in its present stage apply to such data as we get by passive observations? By passive observations we mean observable results of what individuals, firms, etc., actually do in the course of events, not what they might do, or what they think they would do under certain other specified circumstances. (16)

Laws refer to hypothetical situations of what might happen, whereas observations are related that what actually happens. Or in other words, laws are "describing a schedule of alternatives at a given moment, before any particular decision [on what to do] has been taken," whereas the relations we find by regression analysis are "those intended to describe what the individuals actually do at any time" (18).

To acquire knowledge about laws, we need to acquire knowledge about the potential influences that are accounted for in a law. Each potential influence significantly different from zero is a relevant causal factor. To gain this knowledge, Haavelmo distinguishes two different classes of experiments:

(1) experiments that we should like to make to see if certain real economic phenomena-when artificially isolated from "other influences"would verify certain hypotheses, and (2) the stream of experiments that Nature is steadily turning out from her own enormous laboratory, and which we merely watch as passive observers. (14)

In case of the first class of experiments, to isolate a selected set of factors, $x_{1}, \ldots, x_{n}$, from the other influences, $x_{n+1}, x_{n+2}, \ldots$, we first impose ceteris paribus conditions on the latter set $-\Delta x_{n+1}=\Delta x_{n+2}=\ldots=0$ - so that a simpler relationship can be investigated:

$$
\Delta y_{C P}=\frac{\partial f}{\partial x_{1}} \Delta x_{1}+\ldots+\frac{\partial f}{\partial x_{n}} \Delta x_{n}
$$


In such a controlled experiment, the remaining factors, $x_{i}$, can be varied in a systematic way to gain knowledge about the $\partial f / \partial x_{i}$ 's and so to see whether they are stable for these variations. If this applies for all factors $x_{1}, \ldots, x_{n}$, we have found a lawful relation between $y$ and these factors.

In the second class of experiments, we usually passively observe a limited number of factors that have a nonnegligible factual influence

$$
\begin{gathered}
\left(\frac{\partial f}{\partial x_{n+1}} \Delta x_{n+1} \approx \frac{\partial f}{\partial x_{n+2}} \Delta x_{n+2} \approx \ldots \approx 0\right): \\
\Delta y_{P O} \approx \frac{\partial f}{\partial x_{1}} \Delta x_{1}+\ldots+\frac{\partial f}{\partial x_{n}} \Delta x_{n} .
\end{gathered}
$$

Thus, the relationship $y=f\left(x_{1}, \ldots, x_{n}\right)$ explains the actual observed values of $y$, provided that the other factual influences of all the unspecified factors are negligible.

The problem with passive observations, however, is that it is not possible to identify the reason for the factual influence of a factor, say, $x_{n+1}$, being negligible, $\frac{\partial f}{\partial x_{n+1}} \Delta x_{n+1} \approx 0$. We cannot distinguish whether its potential influence is very small, $\frac{\partial f}{\partial x_{n+1}} \approx 0$, or whether the factual variation of this factor during the period for which the data apply was too small, $\Delta x_{n+1} \approx 0$. We would like only to omit the factors whose influence was not observed because their potential influence was negligible to start with. At the same time, we want to retain factors whose influence was not observed because they varied so less that their potential influence was veiled, but may “explain apparent 'breaks in structure' later" (Haavelmo 1944, 26).

To know whether a certain factor is a potential influence, and should therefore be taken account of, we are dependent on Nature's experiments. Regression analysis is based on Nature's variations and therefore does not give knowledge about whether a set of theoretically suggested causal factors is complete.

This problem of passive observation can be tackled in two ways. One is to accumulate data sets with the aim that an increasing number of potential influences become visible; the other is to take account of as many causal factors as theories suggest.

Frequently, our greatest difficulty in economic research does not lie in establishing simple relations between actual observation series, but rather in the fact that the observable relations, over certain time intervals, appear to be still simpler than we expect them to be from theory, so that we are thereby led to throw away elements of a theory that 
would be sufficient to explain apparent "breaks in structure" later. (Haavelmo 1944, 26)

How can this framework help us to "establish constant laws of economic life," where one has to deal with "ever-shifting 'environments"" (17)? Haavelmo's framework shows that besides ceteris paribus environments, laws can also exist in ceteris neglectis environments. These are environments in which all kinds of background influences are constantly changing, but the "permanence" of a law is not affected because the potential background influences are all negligible, $\partial f / \partial x_{n+1} \approx \partial f / \partial x_{n+2} \approx \ldots \approx 0$.

\section{Conclusions}

The discussion of Marshall's problem-whether economics is an exact science-was recently revived by John Sutton (2000) in his Gaston Eyskens Lecture with the telling title, "Marshall's Tendencies: What Can Economists Know?" According to Sutton, "If the analogy of the tides were valid in economics, life would be much easier for economists" (5). The reason for this is that in the early 1950s, a successful program, "the standard paradigm of applied economics," was designed, and it worked perfectly for phenomena like tides. "If Marshall's analogy were valid, we would have seen spectacular progress in economics over the past fifty years" (5). According to Sutton, this standard paradigm is the econometric program set out by Haavelmo in his Probability Approach in Econometrics.

Sutton sees this "standard paradigm" (a science of the tides) as consisting of three properties:

(1) The true model captures a "complete" set of factors that exert large and systematic influences,

(2) All remaining influences can be treated as a noise component that can be modeled as a draw from some probability distribution, and

(3) The model determines a unique equilibrium. (Sutton 2000, 20)

Although this paradigm, according to Sutton, works successfully for tide phenomena, it has failed for economic phenomena. Suppose there is a true model linking an endogenous variable $y$ to a vector $\mathbf{x}=\left(x_{1}, \ldots\right.$, $x_{n}$ ) of exogenous variables, in the sense that

$$
y_{i}=a_{1} x_{i, 1}+a_{2} x_{i, 2}+\ldots+a_{n} x_{i, n}+\eta_{i}(i=1, \ldots, m) .
$$


"I want to take away the sharp distinction between two different types of influence, which we had in the tides analogy, where the astronomical components played the role of the $x_{i}$ 's and the meteorological components played the role of the noise component, $\eta_{i}$ " (21). In practice, many of the $x$ 's may be difficult to measure, even by way of some proxy variable that we might use to control for their effects. "We are stuck with the fact that some of our systematic influences have slipped into our estimated 'residuals,' that is, into the noise component" (21).

I believe that much of the difficulty economists have encountered over the past fifty years can be traced to the fact that the economic environments we seek to model are sometimes too messy to be fitted into the mold of a well-behaved, complete model of the standard kind. It is not generally the case that some sharp dividing line separates a set of important systematic influences that we can measure, proxy, or control for, from the many small unsystematic influences that we can bundle into a "noise" term. (32)

The article I have written here has shown that this "standard paradigm" was not Haavelmo's.

Haavelmo knew the limitations of the standard paradigm too well:

Certainly we know that decisions to consume, to invest, etc., depend on a great number of factors, many of which cannot be expressed in quantitative terms. What is then the point of trying to associate such behavior with only a limited set of measurable phenomena, which cannot give more than an incomplete picture of the whole "environment" or "atmosphere" in which the economic planning and decisions take place? (Haavelmo 1944, 3)

The epistemological framework Haavelmo developed to answer this question was much more refined than Sutton suggests. This epistemology is to be found in the first two chapters of his Probability Approach, which actually took account of the limitations of the tide analogy: Let us assume that for each individual, her consumption is explained by the equation

$$
y^{*}=f\left(x_{1}, x_{2}, \ldots, x_{n}\right) .
$$

However, if we do this for each individual, we shall find-no matter what be the fixed function $f$-that our "explanation" is incomplete. More specifically, we shall find that two individuals, or the same individual in 
two different time periods, may be confronted with exactly the same set of specified influencing factors $x$ (and, hence, they have the same $y^{*}$ [by the equation given just above]), and still the two individuals may have different quantities $y$, neither of which may be equal to $y^{*}$. We may try to remove such discrepancies by introducing more "explaining factors," $x$. But usually, we shall soon exhaust the number of factors which could be considered as common to all individuals, and which, at the same time, were not merely of negligible influence upon $y$. The discrepancies $y-y^{*}$ for each individual may depend upon a great variety of factors, these factors may be different from one individual to another, and they may vary with time for each individual. (Haavelmo 1944, 50)

Current philosophers of science, like Nancy Cartwright (1999), believe that the laws of nature only occur where circumstances are similar, that is, in ceteris paribus environments. If they are right, the possibility of a scientific economics would be problematic. Haavelmo believed the "crazy" opposite, expressed in the epigraph from Cartwright. Although he did not believe that economics could be turned into an exact science, he did not conclude that we should give up our "hope to find elements of invariance in economic life, upon which to establish permanent 'laws"' (Haavelmo 1944, 13). Haavelmo's framework "may give us some hint as to how optimistic or pessimistic we have reason to be: we can try to indicate what would have to be the actual situation in order that there should be no hope of establishing simple and stable causal relations" (23). It shows that for the existence of laws we do not need to have the hard requirement of ceteris paribus environments, but we can do with the softer requirement of a ceteris neglectis environment.

This does not deny the other part of the problem of finding laws by passive observations, namely, how to gain knowledge about potential influences, which ones are relevant and stable. Theories can help- "it is a task of making fruitful hypotheses as to how reality actually is" (Haavelmo 1944, 31) - and the accumulation of data sets may also help (see also Sutton 2000, 21). Nevertheless,

it is a creative process, an art, operating with rationalized notions of some real phenomena and of the mechanism by which they are produced. The whole idea of such models rests upon a belief, already backed by a vast amount of experience in many fields, in the existence of certain elements of invariance in a relation between real phenomena, provided we succeed in bringing together the right ones. (Haavelmo 1944, 10) 


\section{References}

Bjerkholt, O. 2005. "Frisch's Econometrics Laboratory and the Rise of Trygve Haavelmo's Probability Approach." Econometric Theory 21:491-533.

Bjerkholt, O., and D. Qin, eds. 2011. A Dynamic Approach to Economic Theory: Lectures by Ragnar Frisch at Yale University. London: Routledge.

Boumans, M. 2010. "The Problem of Passive Observation." History of Political Economy 42 (1): 75-110.

Cartwright, N. 1999. The Dappled World: A Study of the Boundaries of Science. Cambridge: Cambridge University Press.

Frisch, R. (1930) 2011. “The Yale Lectures.” In Bjerkholt and Qin 2011. 1934. Statistical Confluence Analysis by Means of Complete Regression Systems. Oslo: Universitetets Økonomiske Institutt.

Haavelmo, T. 1938. "Drei Beispiele der ökonometrischen Forschung in den Niederlanden." Weltwirtschaftliches Archiv 48:7*-11*.

—_. (1939) 2008. "On the Statistical 'Testing' of Hypotheses in Economic Theory.” Translated by Erik Biørn. Unpublished lecture. www.sv.uio.no/econ/english /research/networks/haavelmo-network/.

- 1941a. "The Effect of the Rate of Interest on Investment: A Note." Review of Economic Statistics 23 (1): 49-52.

1941b. On the Theory and Measurement of Economic Relations. Unpublished document. www.sv.uio.no/econ/english/research/networks/haavelmo-network/.

- 1944. The Probability Approach in Econometrics. Supplement to Econometrica 12.

Koopmans, T. 1937. Linear Regression Analysis of Economic Time Series. Netherlands Economic Institute, Publication No. 20. Haarlem: Bohn.

Marshall, A. (1890) 1930. Principles of Economics. 8th ed. London: Macmillan.

Morgan, M. S. 1990. The History of Econometric Ideas. Cambridge: Cambridge University Press.

Mill, J. S. (1843) 1911. A System of Logic: Ratiocinative and Inductive. 8th ed. London: Longmans, Green and Co.

Sutton, J. 2000. Marshall's Tendencies: What Can Economists Know? Leuven: Leuven University Press.

Tinbergen, J. 1939a. Statistical Testing of Business-Cycle Theories. Vol. 1, A Method and Its Application to Investment Activity. Geneva: League of Nations.

- 1939b. Statistical Testing of Business-Cycle Theories. Vol. 2, Business Cycles in the United States of America, 1919-1932. Geneva: League of Nations. Wilson, E. B. 1946. Review of The Probability Approach in Econometrics, by Trygve Haavelmo. Review of Economics and Statistics 28 (3): 173-74. 
Published by Duke University Press 